\begin{tabular}{|c|l|}
\hline Title & $\begin{array}{l}\text { Renormalization of the highly forward-peaked phase function using the double exponential formula for radiative } \\
\text { transfer }\end{array}$ \\
\hline Author(s) & Fujii, Hiroyuki; Okawa, Shinpei; Y amada, Y ukio; Hoshi, Y oko; W atanabe, Masao \\
\hline Citation & $\begin{array}{l}\text { Journal of Mathematical Chemistry, 54/10), 2048-2061 } \\
\text { https://doi.org/10.1007/310910-016-0670-3 }\end{array}$ \\
\hline Issue Date & 2016-11 \\
\hline Doc URL & http://hdl.handle.net/2115/67483 \\
\hline Rights & The final publication is available at Springer via http://dx.doi.org/10.1007/s10910-016-0670-3 \\
\hline Type & article (author version) \\
\hline File Information & JMC_Fujii.pdf \\
\hline
\end{tabular}

Instructions for use 


\title{
Renormalization of the highly forward-peaked phase function using the double exponential formula for radiative transfer
}

\author{
Hiroyuki Fujii · Shinpei Okawa · Yukio \\ Yamada • Yoko Hoshi • Masao Watanabe
}

Received: date / Accepted: date

\begin{abstract}
Numerical calculation of photon migration in biological tissue using the radiative transfer equation (RTE) has attracted great interests in biomedical optics and imaging. Because biological tissue is a highly forward-peaked scattering medium, renormalization of the phase function in numerical calculation of the RTE is crucial. This paper proposes a simple approach of renormalizing the phase function by the double exponential formula, which was heuristically modified from the original one. Firstly, the validity of the proposed approach was tested by comparing numerical results for an average cosine of the polar scattering angle calculated by the proposed approach with those by the conventional approach in highly forward-peaked scattering. The results show that calculation of the average cosine converged faster using the proposed approach than using the conventional one as a total number of discrete angular directions increases. Next, the accuracy of the numerical solutions of the RTE using the proposed approach was examined by comparing the numerical solutions with the analytical solutions of the RTE in a homogeneous medium with highly forward-peaked scattering. It was found that the proposed approach reduced the errors of the numerical solutions from those using the conventional one especially at a small value of the total number of the directions. This result suggests that the proposed approach has a possibility to
\end{abstract}

\section{H. Fujii}

Division of Mechanical and Space Engineering, Faculty of Engineering, Hokkaido University, Kita 13 Nishi 8, Kita-ku, Sapporo, Hokkaido 060-8628, Japan

E-mail: fujii-hr@eng.hokudai.ac.jp

S. Okawa

National Defense Medical College, 3-2 Namiki, Tokorozawa, Saitama, 359-8513 Japan

Y. Yamada

Brain Science Inspired Life Support Research Center, University of Electro-Communications, 1-5-1 Chofugaoka, Chofu, Tokyo 182-8585, Japan

Y. Hoshi

Preeminent Medical Photonics Education \& Reseach Center, Hamamatsu University School of Medicine, 1-20-1 Handayama, Higashi-ku, Hamamatsu, Sizuoka 431-3192, Japan

M. Watanabe

Division of Mechanical and Space Engineering, Faculty of Engineering, Hokkaido University, Kita 13 Nishi 8, Kita-ku, Sapporo, Hokkaido 060-8628, Japan 
improve the accuracy for the numerical results of the RTE in the highly scattering medium.

Keywords Radiative transfer equation - renormalization approach of the phase function $\cdot$ highly forward-peaked scattering media $\cdot$ double-exponential formula

Mathematics Subject Classification (2000) 85A25 -92C55 - 65D30 - 74E10

\section{Introduction}

Numerical calculation of photon migration in biological tissue using the radiative transfer equation (RTE) is crucial in biomedical optics and imaging such as diffuse optical tomography (DOT) [10]. DOT has a possibility to enable in-vivo imaging of various organs and tissue volumes because the imaging technique non-invasively reconstructs spatial distributions of physiological and structural information in organs and tissue volumes by using the near-infrared light in the wave length range from $700 \mathrm{~nm}$ to $1000 \mathrm{~nm}$ based on the relationship between the absorption coefficient and a concentration of chemical components (oxy- and deoxy hemoglobins); and between the scattering coefficient and structural properties. In the wave length range, scattering of photon is dominant over absorption of photon [5], so that photons migrate in multi-path. Due to this fact, a conventional image reconstruction algorithm for X-ray computed tomography is not applicable, where absorption of $\mathrm{X}$-ray is dominant over scattering of X-ray. Hence, DOT requires a mathematical model to describe photon migration in biological tissue.

The RTE can accurately describe photon migration in scattering media such as biological tissue in terms of the light intensity as a function of position, angular direction, and time. Also, the RTE has a scattering integral of the light intensity multiplied by the phase function over the whole directions, which expresses the energy incoming to the position of interest from all the directions by scattering. Because analytical solutions of the RTE are obtained for the limited cases of simple geometries such as infinite and homogeneous media [22], the RTE is approximated to simpler formulations like the simplified spherical harmonics system $[26,12]$ or numerically solved for complicated geometries of organs and tissue volumes. In the numerical calculation of the RTE, the independent variables (position, direction, and time) are discretized by numerical schemes. For discretization of the direction, the discrete ordinates method (DOM) has been widely used [4], and for spatial and temporal discretization, the finite difference, finite volume, or finite element methods are employed. Then, the integro-differential equation of the RTE is formulated to a set of algebraic equations.

In the DOM, a quadrature set of the discrete angular directions and weights should be chosen appropriately for fast convergence of the quadrature sum and for high accuracy of the numerical solution of the RTE. Although various quadrature sets for the DOM have been proposed $[3,6,1]$, the most common choice of the sets is the level symmetric even (LSE) set [6], which is determined to satisfy the symmetry to all directions over the unit sphere. It has been shown that numerical calculation of the RTE using the LSE set is accurate especially for isotropic scattering as in the case of neutron transport. However, it has been reported that in highly forwardpeaked scattering media such as biological tissue, the numerical solution of the 
RTE with the LSE set is inaccurate $[15,2]$. This is because in highly forwardpeaked scattering, the value of the phase function of the RTE at the small polar scattering angle tends to increase exponentially, resulting in slow convergence of the numerical calculation of the scattering integral. For the quadrature set, the extended trapezoidal rule (ETR) has been employed $[16,19]$. The advantage of the ETR set is that the total number of the directions can be chosen according to a computer memory size, so that the numerical calculation of the scattering integral can converge with a sufficiently large number of the directions and enough computer memory size. However, it leads to high computational loads for numerical calculations of the RTE.

To overcome the difficulty stated above, several works have proposed approaches to renormalize the phase function for highly forward-peaked scattering in the filed of radiative heat transfer $[17,2,14]$. At the early stage, Liu and coworkers have renormalized the phase function by an inverse of the quadrature sum of the phase function over the whole directions [17]. Despite the simple approach, they have shown that the accuracy of the numerical results of the RTE is improved because their approach satisfies the normalization condition of the phase function over the whole directions. In their approach, however, numerical results of the average cosine of the polar scattering angle are deviated from the anisotropic factor, to which the average cosine must be equal. To satisfy the condition for the average cosine stated above, recently, the phase function has been renormalized by weight matrices calculated using the finite volume method [2] or an inverse analysis [14]. It has been actively reported that the renormalization approaches can provide the accurate numerical results. However, a further development of the renormalization approach is still necessary to improve the accuracy of the numerical results.

This paper proposes a simple approach to renormalize the phase function in highly forward-peaked scattering by the double exponential formula, which has been originally developed for fast convergence of numerical integration having a singular function $[25,20]$. For validation of the proposed approach, we compared the numerical results of the average cosine calculated using the proposed approach with those using the conventional approach (Liu's renormalization approach [17]). In addition, the proposed approach was validated by comparing the numerical solution of the time-dependent RTE using the proposed approach with an analytical solution of the RTE for a three dimensional, homogeneous, and highly forward-peaked scattering medium.

The following section provides an explanation of the proposed approach for numerical calculation of the time-dependent RTE for a three dimensional scattering medium. Section 3 provides the numerical results to test the validity of the proposed approach. Finally, conclusions are described. 


\section{Photon migration model}

2.1 Radiative transfer equation

The time-dependent RTE can provide an accurate description of photon migration in scattering media, which is formulated in three dimensions (3D) as [4],

$$
\begin{aligned}
& {\left[\frac{\partial}{v \partial t}+\boldsymbol{\Omega} \cdot \nabla+\mu_{a}(\boldsymbol{r})+\mu_{s}(\boldsymbol{r})\right] I(\boldsymbol{r}, \boldsymbol{\Omega}, t)} \\
& =\mu_{s}(\boldsymbol{r}) \int_{4 \pi} d \boldsymbol{\Omega}^{\prime} p\left(\boldsymbol{\Omega} \cdot \boldsymbol{\Omega}^{\prime}\right) I\left(\boldsymbol{r}, \boldsymbol{\Omega}^{\prime}, t\right)+q(\boldsymbol{r}, \boldsymbol{\Omega}, t),
\end{aligned}
$$

where $I(\boldsymbol{r}, \boldsymbol{\Omega}, t)$ in $\mathrm{Wcm}^{-2} \mathrm{sr}^{-1}$ represents the light intensity as a function of spatial location $\boldsymbol{r}=(x, y, z)$ in $\mathrm{cm}$ for a 3D Cartesian coordinate system, angular direction $\boldsymbol{\Omega}=\left(\Omega_{x}, \Omega_{y}, \Omega_{z}\right)=(\sin \theta \cos \phi, \sin \theta \sin \phi, \cos \theta)$ (polar angle, $\theta \in[0, \pi]$, azimuthal angle, $\phi \in[0,2 \pi]$ in sr), and time $t$ in ps. $\mu_{a}(\boldsymbol{r})$ and $\mu_{s}(\boldsymbol{r})$ in $\mathrm{cm}^{-1}$ are the absorption and scattering coefficients, respectively, $v$ is the speed of light in the medium, $p\left(\boldsymbol{\Omega} \cdot \boldsymbol{\Omega}^{\prime}\right)$ in $\mathrm{sr}^{-1}$ is the phase function with $\boldsymbol{\Omega}^{\prime}$ and $\boldsymbol{\Omega}$ denoting the incident and scattered directions, respectively, and $q(\boldsymbol{r}, \boldsymbol{\Omega}, t)$ in $\mathrm{W} \mathrm{cm}^{-2} \mathrm{sr}^{-1}$ is a source function. Here, the phase function is assumed to be not dependent on the azimuthal angle, $\phi$, but to be dependent on the polar scattering angle, $\varphi=\theta-\theta^{\prime}=\cos ^{-1} \boldsymbol{\Omega} \cdot \boldsymbol{\Omega}^{\prime}$, which is the angle between the incident and scattered directions.

\subsection{Henyey-Greenstein phase function}

For the formulation of $p\left(\boldsymbol{\Omega} \cdot \boldsymbol{\Omega}^{\prime}\right)$, the Henyey-Greenstein phase function [13] is widely employed,

$$
p\left(\boldsymbol{\Omega} \cdot \boldsymbol{\Omega}^{\prime}\right)=\frac{1}{4 \pi} \frac{1-g^{2}}{\left(1+g^{2}-2 g \boldsymbol{\Omega} \cdot \boldsymbol{\Omega}^{\prime}\right)^{3 / 2}} .
$$

Here, $g$ is the anisotropic factor representing anisotropy of scattering and is defined as the average cosine of the polar scattering angle with the weight of the phase function;

$$
g=\frac{\int_{4 \pi} d \boldsymbol{\Omega}^{\prime} p\left(\boldsymbol{\Omega} \cdot \boldsymbol{\Omega}^{\prime}\right) \boldsymbol{\Omega} \cdot \boldsymbol{\Omega}^{\prime}}{\int_{4 \pi} d \boldsymbol{\Omega}^{\prime} p\left(\boldsymbol{\Omega} \cdot \boldsymbol{\Omega}^{\prime}\right)} .
$$

A case of $g=0$ corresponds to isotropic scattering and a case of $g>0$ to forwardpeaked scattering. It is well known that biological tissue is a highly forward-peaked scattering medium, whose $g$-value is typically larger than 0.8 [5].

At any values of $g$, the following normalization conditions for the phase function and for the average cosine must be satisfied,

$$
\begin{aligned}
& \int_{4 \pi} d \boldsymbol{\Omega}^{\prime} p\left(\boldsymbol{\Omega} \cdot \boldsymbol{\Omega}^{\prime}\right)=1, \\
& g^{-1} \int_{4 \pi} d \boldsymbol{\Omega}^{\prime} p\left(\boldsymbol{\Omega} \cdot \boldsymbol{\Omega}^{\prime}\right) \boldsymbol{\Omega} \cdot \boldsymbol{\Omega}^{\prime}=1 .
\end{aligned}
$$




\section{Numerical schemes for the time-dependent RTE}

In this study, the time-dependent RTE is numerically solved based on the DOM for angular direction and on the finite-difference method for space and time.

\subsection{Discrete ordinates method}

The DOM approximates the scattering integral in Eq. (1) to a quadrature sum:

$$
\mu_{s}(\boldsymbol{r}) \int_{4 \pi} d \boldsymbol{\Omega}^{\prime} p\left(\boldsymbol{\Omega} \cdot \boldsymbol{\Omega}^{\prime}\right) I\left(\boldsymbol{r}, \boldsymbol{\Omega}^{\prime}, t\right) \sim \mu_{s}(\boldsymbol{r}) \sum_{l^{\prime}=1}^{N_{\Omega}} w_{l^{\prime}} p_{l l^{\prime}} I_{l^{\prime}}\left(\boldsymbol{r}, \boldsymbol{\Omega}_{l^{\prime}}, t\right)
$$

where $w_{l^{\prime}}$ is a weight for numerical integration, $\Omega_{l^{\prime}}$ is the $l^{\prime}$-th discrete angular direction, and $N_{\Omega}$ is a total number of the discrete angular directions. In the DOM, a quadrature set of $\left(w_{l}, \boldsymbol{\Omega}_{l}\right)$ needs to be chosen appropriately. Here, we consider the two kinds of quadrature sets: the LSE [6] and ETR [16] sets. Although efficient schemes for numerical integration have been extensively developed by modifying the conventional closed Newton-Cotes method [24], we consider the conventional method (ETR), which is one of the closed Newton-Cotes methods. The LSE set is determined to satisfy the symmetry to all the directions over the unit sphere because photons are scattered in all the directions and numerical solutions of the RTE should be invariant with respect to a 90 degree axis rotation and a line reflection as shown in Figs. 1(a) and (b). However, a shortcoming of the LSE set is a limitation of $N_{\Omega}$; a maximum value of $N_{\Omega}$ is 288 (termed as $S_{16}$ ) under a condition that $w_{l}$ is positive. Although for highly forward-peaked scattering, a larger value of $N_{\Omega}$ is necessary for convergence of the numerical integration (Eq. (6)), the maximum value of $N_{\Omega}$ for the LSE set is insufficient.

The ETR is a simple method to provide the set of $\left(w_{l}, \Omega_{l}\right)$ and $N_{\Omega}$ can be chosen by a computer memory size. Hence, the numerical integration using the ETR for highly forward-peaked scattering can be converged when $N_{\Omega}$ is sufficiently large. Although the directions by the ETR are symmetric over the $x-y$ plane (Fig. $1(\mathrm{c})$ ), however, they are not symmetric over the $y-z$ and $z-x$ planes (Fig. $1(\mathrm{~d})$ ).

\subsection{Renormalization approaches of the phase function}

Figure 2 shows the Henyey-Greenstein phase function (Eq. (2)) as a function of $\boldsymbol{\Omega} \cdot \boldsymbol{\Omega}^{\prime}$ for the cases of $g=0.0,0.5$, and 0.9 , in logarithmic scale. For the isotropic scattering $(g=0.0)$, the value of the phase function is constant over the whole region from $\boldsymbol{\Omega} \cdot \boldsymbol{\Omega}^{\prime}=-1(\varphi=\pi)$ to $\boldsymbol{\Omega} \cdot \boldsymbol{\Omega}^{\prime}=1(\varphi=0)$, meanwhile, for the highly forward-peaked scattering $(g=0.9)$ the value of the phase function tends to increase exponentially as $\boldsymbol{\Omega} \cdot \boldsymbol{\Omega}^{\prime}$ approaches to unity ( $\varphi$ to 0 ). This fact means that for fast convergence of the numerical integration, the values of the weight, $w_{l^{\prime}}$, should be dependent on $\boldsymbol{\Omega}_{l} \cdot \Omega_{l^{\prime}}$. However, the values of $w_{l^{\prime}}$ is determined to satisfy the symmetry over the unit sphere like the LSE set. As a result, the normalization conditions of Eqs. (4) and (5) are not satisfied in the discrete forms 


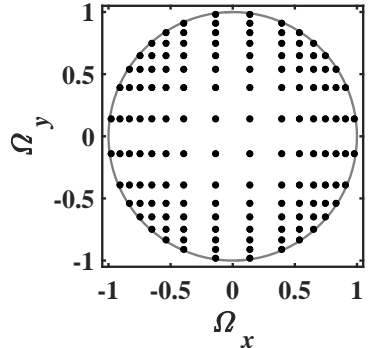

(a) LSE set in $x-y$ plane

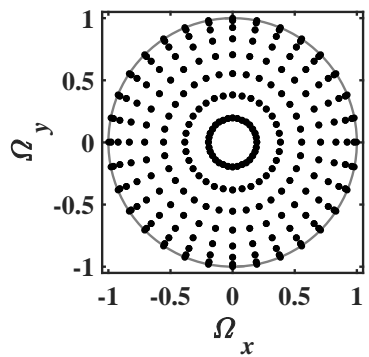

(c) ETR set in $x-y$ plane

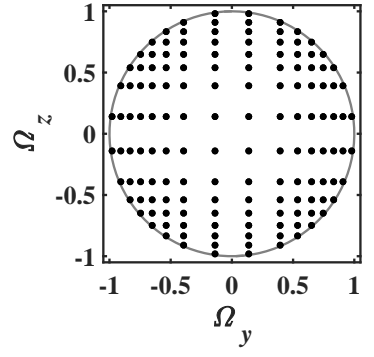

(b) LSE set in $y-z$ plane

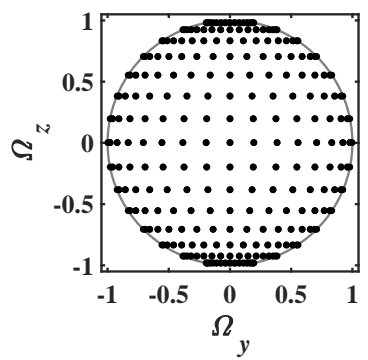

(d) ETR set in $y-z$ plane

Fig. 1 Discrete angular directions in $x-y$ and $y$ - $z$ planes for the LSE set $\left(N_{\Omega}=288\right)$ ((a) and (b)) and ETR set $\left(N_{\Omega}=480\right)((\mathrm{c})$ and $(\mathrm{d}))$. Dots are the discrete angular directions, and circles are boundaries of the unit sphere

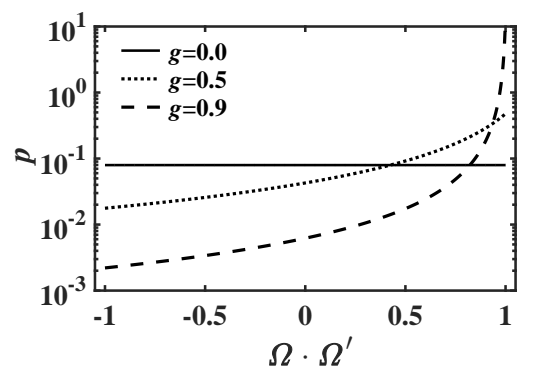

Fig. 2 Henyey-Greenstein phase function as a function of $\Omega \cdot \Omega^{\prime}$ for isotropic scattering $(g=$ $0.0)$, moderately forward-peaked scattering $(g=0.5)$, and highy forward-peaked scattering $(g=0.9)$, in logarithmic scale

for highly forward-peaked scattering media:

$$
\begin{array}{rl}
\sum_{l^{\prime}=1}^{N_{\Omega}} w_{l^{\prime}} p_{l l^{\prime}} \neq 1 & l=1,2, \cdots, N_{\Omega}, \\
g^{-1} \sum_{l^{\prime}=1}^{N_{\Omega}} w_{l^{\prime}} p_{l l^{\prime}} \Omega_{l} \cdot \Omega_{l^{\prime}} \neq 1 & l=1,2, \cdots, N_{\Omega} .
\end{array}
$$

To overcome the problem, Liu and co-workers [26] have proposed the renormalized phase function, $\hat{p}_{l l^{\prime}}$, in the following form by introducing a renormalizing 
factor of $f_{l}$,

$$
\hat{p}_{l l^{\prime}}=f_{l} p_{l l^{\prime}}, \quad f_{l}=\left[\sum_{l^{\prime}=1}^{N_{\Omega}} w_{l^{\prime}} p_{l l^{\prime}}\right]^{-1} .
$$

This simple approach improves the accuracy of the numerical results of the RTE by satisfying the normalization condition for the phase function (Eq. (4)). However, the normalization condition for the average cosine (Eq. (5)) is still unsatisfied.

This paper proposes a simple approach to renormalize the phase function for satisfying both of the normalization conditions in the following equations:

$$
\hat{p}_{l l^{\prime}}=f_{l} W_{l l^{\prime}} p_{l l^{\prime}}, \quad f_{l}=\left[\sum_{l^{\prime}=1}^{N_{\Omega}} w_{l^{\prime}} W_{l l^{\prime}} p_{l l^{\prime}}\right]^{-1} .
$$

A renormalizing matrix $W_{l l^{\prime}}$ is calculated by the double exponential formula (DEF), which has been originally developed for fast convergence of numerical integration of a singular function $[25,20]$,

$$
W_{l l^{\prime}}=\left\{\begin{array}{ll}
\frac{\cosh \left(s_{l l^{\prime}}\right) \exp \left[-2 \sinh \left(s_{l l^{\prime}}\right)\right]}{\left\{\cosh \left[\sinh \left(s_{l l^{\prime}}\right)\right] \exp \left[-\sinh \left(s_{l l^{\prime}}\right)\right]\right\}^{2}} & \left|s_{l l^{\prime}}\right| \leq s_{\max } \\
\frac{\cosh \left(s_{\max }\right) \exp \left[-2 \sinh \left(s_{\max }\right)\right]}{\left\{\cosh \left[\sinh \left(s_{\max }\right)\right] \exp \left[-\sinh \left(s_{\max }\right)\right]\right\}^{2}} & \left|s_{l l^{\prime}}\right|>s_{\max }
\end{array},\right.
$$

where $s_{l l^{\prime}}=\sinh ^{-1}\left(\tanh ^{-1}\left(\Omega_{l} \cdot \Omega_{l^{\prime}}\right)\right)$; and $s_{\max }$ is a maximum value of $s_{l l^{\prime}}$ and is the only fitting parameter in the proposed approach. Using the LevenbergMarquardt method, $s_{\max }$ is determined so that the normalized average cosine of $g^{-1} \sum_{l^{\prime}=1}^{N_{\Omega}} w_{l^{\prime}} \hat{p}_{l l^{\prime}} \boldsymbol{\Omega}_{l} \cdot \boldsymbol{\Omega}_{l^{\prime}}$ converges to unity. Although the set of $\left(w_{l}, \boldsymbol{\Omega}_{l}\right)$ is calculated by the DEF with an unequal step size, our proposed approach utilizes the set given by the ETR. This is because the directions by the DEF are not symmetric over the unit sphere and inappropriate. In that sense, here, the DEF is heuristically modified.

\subsection{Finite difference method}

The 3rd order upwind scheme is employed for spatial discretization and the 3rd order TVD (total variation diminishing)-Runge-Kutta method [11] is employed for temporal discretization, respectively. For time integration, modifications of the Runge-Kutta type method (Runge-Kutta-Nyström method) or numerically efficient schemes have been extensively proposed [23,21]. Nevertheless, we employ the conventional TVD Runge-Kutta scheme among the methods because here we focus on the renormalization of the phase function over time integration. In the finite difference method, $x, y, z$, and $t$ are discretized as $x_{i}=i \Delta x(i \in$ $\left.\left\{0, \cdots, N_{x}\right\}\right), y_{j}=j \Delta y\left(j \in\left\{0, \cdots, N_{y}\right\}\right), z_{k}=k \Delta z\left(k \in\left\{0, \cdots, N_{z}\right\}\right)$, and $t_{m}=m \Delta t\left(m \in\left\{0, \cdots, N_{t}\right\}\right)$ with the constant step sizes of $\Delta x, \Delta y, \Delta z$, and $\Delta t$, respectively, and the numbers of the grid nodes and timesteps of $N_{x}, N_{y}, N_{z}$, and $N_{t}$, respectively. The step sizes are $\Delta x=\Delta y=\Delta z=0.08 \mathrm{~cm}$ and $\Delta t=0.5$ ps, respectively. In our preliminary study, it was confirmed that the numerical solutions of the RTE are unchanged even as the step sizes are finer than those given above. 
Table 1 Quadrature sets of $\left(w_{l}, \Omega_{l}\right)$ and renormalization approaches

\begin{tabular}{lcc}
\hline & $\left(w_{l}, \boldsymbol{\Omega}_{l}\right)$ & $\hat{p}_{l l^{\prime}}$ \\
\hline Method A (proposed here) & ETR & DEF (Eqs. (10) and (11)) \\
Method B & ETR & Liu's approach (Eq. (9)) \\
Method C & LSE & Liu's approach (Eq. (9)) \\
\hline
\end{tabular}

\subsection{Numerical conditions}

To test the validity of the proposed approach, three methods for the quadrature sets, $\left(w_{l}, \boldsymbol{\Omega}_{l}\right)$, and for the renormalization approaches of phase functions, $\hat{p}_{l l^{\prime}}$, are compared in Table 1, Method A (proposed here): $\left(w_{l}, \boldsymbol{\Omega}_{l}\right)$ is given by the ETR set and $\hat{p}_{l l^{\prime}}$ is calculated by the DEF (Eqs. (10) and (11)), Method B: $\left(w_{l}, \boldsymbol{\Omega}_{l}\right)$ by the ETR set and $\hat{p}_{l l}$, by the Liu's approach (Eq. (9)), and Method C: $\left(w_{l}, \boldsymbol{\Omega}_{l}\right)$ by the LSE set and $\hat{p}_{l l^{\prime}}$ by the Liu's approach.

This paper examines convergence of the average cosine as $N_{\Omega}$ increases, and compares the numerical solutions of the RTE using the three methods with the analytical solutions for the case of $g=0.9$ which is a typical value for biological tissue. For Methods A and B using the LSE set, $N_{\Omega}$ are varied at $60,112,180$, 264, 364, 480, 760, 1104, and 1512; and for Method C using the LSE set, 48, 80, 168 , and 288 .

The source code for the numerical calculations were written in the C programming language, and all the matrices were compressed to vectors as the compressed row storage format. Also, parallel CPU programming was implemented with 48 thread computers (Intel Xeon E5-2690v3@2GHz) by using the OpenMP, which is a portable and shared-memory programming scheme.

\section{Numerical results}

4.1 Normalization condition for the average cosine

Firstly, we examined how the normalization for the average cosine, Eq. (5), is satisfied as stated above. Here, the normalization condition of the phase function, Eq. (4), was confirmed to be satisfied by the three methods automatically due to the renormalizing factor, $f_{l}$ (Eq. (10) for Method A; and Eq. (9) for Methods B and C). For the examination of the average cosine, we calculate numerical integration of $S_{l}^{g}$ as expressed in the following,

$$
S_{l}^{g}=g^{-1} \sum_{l^{\prime}=1}^{N_{\Omega}} w_{l^{\prime}} \hat{p}_{l l^{\prime}} \boldsymbol{\Omega}_{l} \cdot \boldsymbol{\Omega}_{l^{\prime}} \quad l=1,2, \cdots, N_{\Omega} .
$$

Here, $S_{l}^{g}$, is calculated at each integer value of $l$ from 1 to $N_{\Omega}$, and $S_{l}^{g}$ should ideally be unity (Eq. (5)). Figure 3(a) shows the mean values of $S_{l}^{g}$ averaged over $l$, defined as $\bar{S}_{l}^{g}=\sum_{l=1}^{N_{\Omega}} S_{l}^{g} / N_{\Omega}$ at different integer values of $N_{\Omega}$ in a logarithmic scale for the three methods. For Method A (proposed here), the values of $\bar{S}_{l}^{g}$ are almost unity for all the values of $N_{\Omega}$ by adjusting $s_{\max }$ in Eq. (11) so as to satisfy Eq. (5). The adjusted values of $s_{\max }$ are shown in the inset of Fig. 3(a) to 

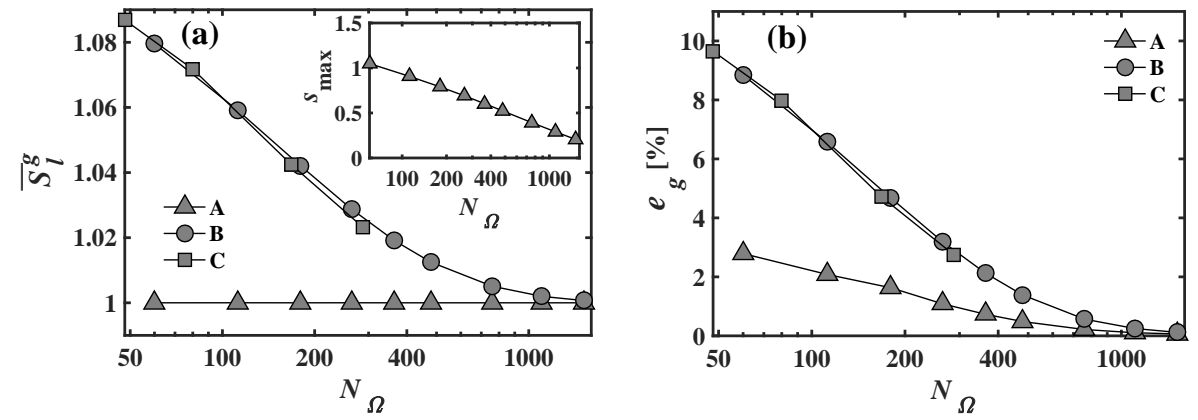

Fig. 3 (a) Mean values of $S_{l}^{g}, \bar{S}_{l}^{g}$, for Methods A, B, and C as listed in Table 1 with $g=0.9$ at different values of $N_{\Omega}$ in a logarithmic scale and $s_{\max }$ (Eq. (11)) is plotted in the inset. (b) Mean absolute percentage error, $e_{g}$ of $S_{l}^{g}$ for the three methods with $g=0.9$.

behave almost linearly to the logarithm of $N_{\Omega}$. The simple linear relation makes the adjusting procedure for $s_{\max }$ easy. It is observed in Fig. 3(a) that the values of $\bar{S}_{l}^{g}$ for Method B converge to unity when $N_{\Omega}$ is larger than approximately 1000. Also, it is found that the dependence of the values of $\bar{S}_{l}^{g}$ for Method C on $N_{\Omega}$ is similar to that for Method B, but $N_{\Omega}$ for the LSE set is limited up to 288 where the minimum of $\bar{S}_{l}^{g}$ is reached at approximately 1.02 .

The deviation of $S_{l}^{g}$ from the unity (theoretical value) is evaluated by the mean absolute percentage error for the average cosine, $e_{g}=\sum_{l=1}^{N_{\Omega}}\left|S_{l}^{g}-1\right| \times 100 / N_{\Omega}$. As shown in Fig. 3(b), the values of $e_{g}$ for Method A are smallest among the three methods, indicating the accuracy of the proposed approach. As well as the results of $\bar{S}_{l}^{g}$, Method B provides the similar results for $e_{g}$ to Method C. These results suggest that for the normalization condition for the average cosine, Eq. (5), the accuracy of the ETR set is the same as that of the LSE set in highly forwardpeaked scattering media, although the LSE set is a higher order than the ETR set.

\subsection{Fluence rate}

In this subsection, we compare the numerical solutions of the RTE using the three methods with the analytical solution of the RTE. As the analytical solution, an approximate RTE solution, $\Phi_{\text {inf }}$, is used here for infinite homogeneous media with highly forward-peaked scattering $[18,22]$,

$$
\begin{aligned}
\Phi_{i n f}(\rho, t) & =v\left(1-\frac{\rho^{2}}{v^{2} t^{2}}\right)^{1 / 8}\left(\frac{3 \mu_{s}^{\prime}}{4 \pi v t}\right)^{3 / 2} G\left(\mu_{s}^{\prime} v t\left[1-\frac{\rho^{2}}{v^{2} t^{2}}\right]^{3 / 4}\right) \\
& \times e^{-\mu_{t}^{\prime} v t} \Theta(v t-\rho), \\
G(\lambda) & =8(3 \lambda)^{-3 / 2} \sum_{\Lambda=1}^{\infty} \frac{\Gamma(3 \Lambda / 4+3 / 2)}{\Gamma(3 \Lambda / 4)} \frac{\lambda^{\Lambda}}{\Lambda !},
\end{aligned}
$$

where $\Phi$ is the fluence rate defined as $\int_{4 \pi} d \Omega I, \Theta$ is the Heaviside step function, $\mu_{t}^{\prime}=\mu_{a}+\mu_{s}^{\prime}, \mu_{s}^{\prime}=(1-g) \mu_{s}$, and $\rho$ is the distance from the source position of $\left(x_{s}, y_{s}, z_{s}\right)$. It has been reported that the approximate solution agrees with 


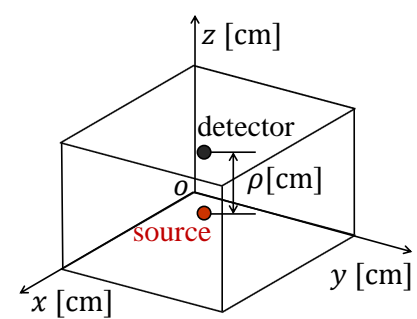

(a)

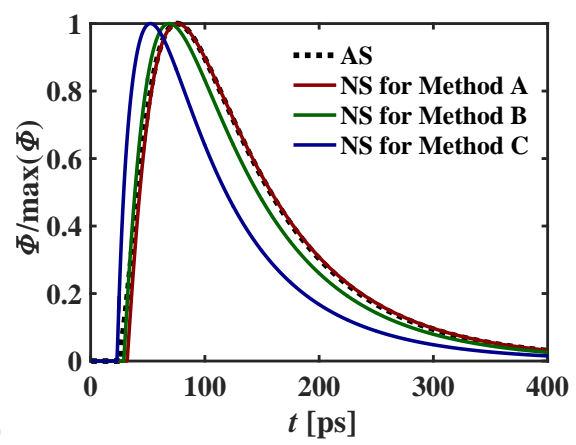

Fig. 4 (a) Source and detector positions in the homogeneous cubic medium. (b) Temporal profiles of the fluence rate, $\Phi$, normalized by their peak values at the source-detector distances, $\rho=0.56 \mathrm{~cm}$ with $g=0.9$. A dotted curve represents the approximate solution (AS) given by Eq. (13); and solid curves represent the numerical solutions (NS) by the three methods listed in Table 1.

Monte Carlo simulations [18]. To compare the analytical solution for infinite media with numerical solutions for finite media, the source and detector are positioned in the finite medium far from the boundary as shown in Fig. 4(a). It has been confirmed that such a comparison test is possible in our previous study $[8,9]$. Here, we consider a homogeneous cubic medium with a side of $2.88 \mathrm{~cm}$, where a source is located at the center of the medium, $\left(x_{s}, y_{s}, z_{s}\right)=(1.44 \mathrm{~cm}, 1.44 \mathrm{~cm}$, $1.44 \mathrm{~cm}$ ) with $\rho=0.56 \mathrm{~cm}$ for the detector position. In our preliminary study, it was confirmed that even when the medium size increases, the numerical solutions are unchanged, meaning little effects of the boundary condition on the numerical solutions. The optical properties of the medium are given as $\mu_{a}=0.38 \mathrm{~cm}^{-1}$, $\mu_{s}=140 \mathrm{~cm}^{-1}$, and $g=0.9$ which are those of the cerebral cortex of a rat at the wave length of $780 \mathrm{~nm}[7]$.

Figure 4(b) shows the temporal profiles of $\Phi_{\text {inf }}$ (Eq. (13)) and numerical solutions for $\Phi$ using the three methods normalized by their peak values at the detector position of $\left(x_{s}, y_{s}, z_{s}+\rho\right)=(1.44 \mathrm{~cm}, 1.44 \mathrm{~cm}, 2.00 \mathrm{~cm})$ with $N_{\Omega}=480$ for Methods A and B, and $N_{\Omega}=288$ for Method C. As shown in Fig. 4(b), the numerical solutions are strongly influenced by the methods of angular discretization although the spatial and temporal discretization are the same. It is observed that the numerical solution using Method A agrees with $\Phi_{\text {inf }}$, while those using Methods B and $\mathrm{C}$ deviate from $\Phi_{\text {inf }}$, meaning that the improvement for the accuracy of the numerical solution was achieved by the proposed approach.

Next, we examined the invariance of the numerical solutions with respect to a 90 degree axis rotation and a line reflection, because it is desirable that the discrete angular directions are symmetric over the unit sphere, and the numerical solutions are invariant with the rotation and reflection. For this examination, we considered six cases of detector positions as shown in Fig. 5(a): $x_{ \pm}=\left(x_{s} \pm\right.$ $\left.\rho, y_{s}, z_{s}\right), y_{ \pm}=\left(x_{s}, y_{s} \pm \rho, z_{s}\right)$, and $z_{ \pm}=\left(x_{s}, y_{s}, z_{s} \pm \rho\right)$ with a source position of $\left(x_{s}, y_{s}, z_{s}\right)$ and source-detector distance of $\rho=0.56 \mathrm{~cm}$. If the numerical solutions satisfy the invariance, the numerical solutions are the same for the six cases. The invariance was evaluated by the mean absolute percentage error, $e_{\Phi}$, of the fluence 
(a)

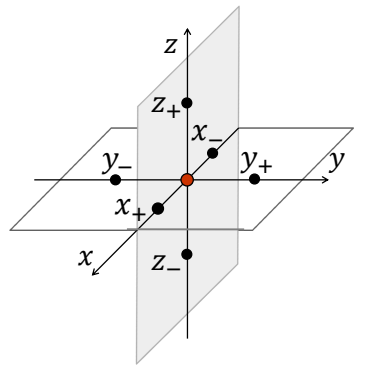

(b)

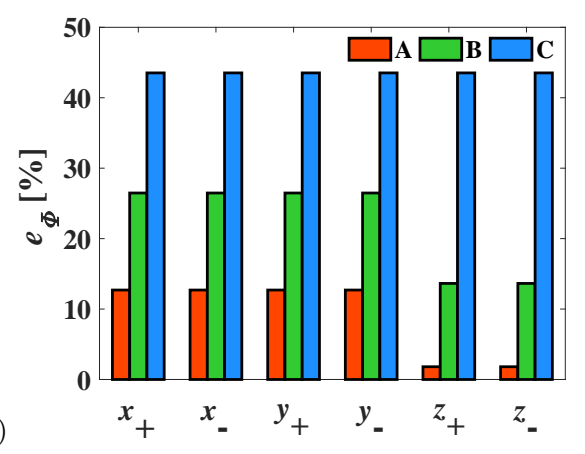

Fig. 5 (a) Six detector positions in the homogeneous medium: $x_{ \pm}=\left(x_{s} \pm \rho, y_{s}, z_{s}\right), y_{ \pm}=$ $\left(x_{s}, y_{s} \pm \rho, z_{s}\right)$, and $z_{ \pm}=\left(x_{s}, y_{s}, z_{s} \pm \rho\right)$. A red dot represents a source position of $\left(x_{s}, y_{s}, z_{s}\right)$. (b) Histogram of $e_{\Phi}$ given by Eq. (14) at the six detector positions for Method A $\left(N_{\Omega}=480\right)$, Method B $\left(N_{\Omega}=480\right)$, and Method $\mathrm{C}\left(N_{\Omega}=288\right)$ at $\rho=0.56 \mathrm{~cm}$. The results at $z_{+}$ correspond to Fig. 4(b).

rate normalized by its peak value, $\hat{\Phi}$,

$$
e_{\Phi}=\frac{1}{N-M} \sum_{m=M}^{N}\left|\frac{\hat{\Phi}^{m}-\hat{\Phi}_{i n f}\left(t_{m}\right)}{\hat{\Phi}_{i n f}\left(t_{m}\right)}\right| \times 100,
$$

where $\hat{\Phi}^{m}$ and $\hat{\Phi}_{i n f}\left(t_{m}\right)$ represent the values of $\hat{\Phi}$ at the $m$-th time step, $t_{m}$, in the numerical and analytical solutions of the RTE, respectively; and the summation with respect to $m$ is over a time period from the peak time $(m=M)$ to the time when $\hat{\Phi}$ reduces to $10^{-1.5} \simeq 0.032(m=N)$. Here, a time period earlier than the peak time is excluded from the calculation of $e_{\Phi}$ because the approximate nature of the analytical solution (Eq. (13)) appears strongly in the early time period, and because in the early time period, the numerical solutions in this study are slightly affected by numerical instability due to the 3rd order upwind scheme.

Figure 5(b) shows a histogram of $e_{\Phi}$ for Method A $\left(N_{\Omega}=480\right)$, Method B $\left(N_{\Omega}=480\right)$, and Method $\mathrm{C}\left(N_{\Omega}=288\right)$ at the six detector positions. Results of $e_{\Phi}$ for Method $\mathrm{C}$ are independent of the detector positions, meaning that Method $\mathrm{C}$ satisfies the invariance with respect to the rotation and reflection. This is because the LSE set is symmetric over the unit sphere as shown in Figs. 1(a) and (b). For Method B, the results of $e_{\Phi}$ are the same for the four detector positions of $x_{ \pm}$and $y_{ \pm}$, indicating the invariance with respect to the rotation and reflection in the $x-y$ plane. However, the results of $e_{\Phi}$ for $x_{ \pm}$and $y_{ \pm}$are different from those for $z_{ \pm}$, meaning a failure of the invariance in the $x-z$ (or $y-z$ ) plane. This is ascribed to the asymmetry of the ETR set over the unit sphere as shown in Figs. 1(c) and (d). Method A reduces $e_{\Phi}$ for all the detector positions from those for Method B. However, Method A less improves the failure of the invariance in the $x-z$ and $y-z$ planes experienced by Method B.

Finally, we calculated $e_{\Phi}$ at different values of $N_{\Omega}$ for the three methods at $\rho=0.56 \mathrm{~cm}$ with $g=0.9$ as shown in Fig. 6 . Among the six detector positions (Fig. 5(a)), here, $x_{-}$and $z_{+}$are selected because $e_{\Phi}$ for Method $\mathrm{C}$ is independent of the positions, and $e_{\Phi}$ for Methods $\mathrm{A}$ and $\mathrm{B}$ at $x_{-}$and $z_{+}$are the same as that at $x_{+}$(or $\left.y_{ \pm}\right)$and $z_{-}$, respectively, as shown in Fig. 5(b). It is observed in Fig. 6 that $e_{\Phi}$ for the three methods decreases as $N_{\Omega}$ increases, and that the values 


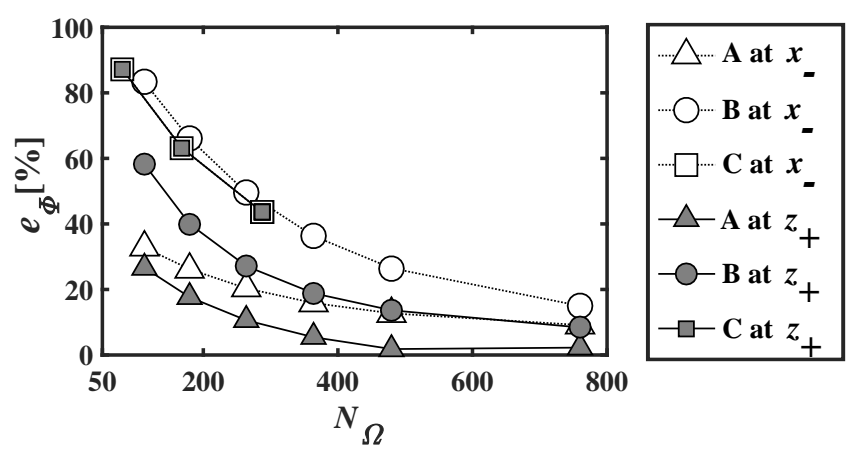

Fig. $6 e_{\Phi}$ for the three methods at different values of $N_{\Omega}$ for $x_{-}$and $z_{+}$at $\rho=0.56 \mathrm{~cm}$ with $g=0.9$. Source-detector positions are the same as Fig. 5(a)

of $e_{\Phi}$ for Method A both at $x_{-}$and $z_{+}$are smaller than those for Method B. In particular, the values of $e_{\Phi}$ for Method A at $z_{+}$are negligibly small in the range of $N_{\Omega}$ larger than around 500. The values of $e_{\Phi}$ for Method C at $x_{-}$(open squares) are hidden behind those at $z_{+}$(solid squares) because they are the same. In the range of $N_{\Omega}$ smaller than about 300, the values of $e_{\Phi}$ for Method B at $x_{-}$(open circles) are similar to those for Method $\mathrm{C}$, indicating that the accuracy of the LSE set (Method C) is almost the same as that of the ETR set (Method B) in the range of $N_{\Omega}$, like the normalization condition of the average cosine discussed in Sec. 4.1. Also, in the range of $N_{\Omega}$ smaller than about 500, the differences of $e_{\Phi}$ between $x_{-}$and $z_{+}$for Method A are smaller than those for Method B, suggesting that the proposed approach is effective in the region of $N_{\Omega}$. As a result, the proposed approach (Method A) can improve accuracy of the numerical solutions of the RTE compared with the conventional methods (Methods A and B) in the whole range of $N_{\Omega}$ in this study.

\section{Conclusions}

In summary, we developed a simple approach for renormalizing the phase function in the RTE based on the double exponential formula. The validity of the proposed approach was confirmed by examining the numerical results of the average cosine and numerical solutions of the RTE for the three methods of the quadrature sets and renormalization approaches. We found that the proposed approach reduced the numerical errors of the average cosine and numerical solutions when compared with the conventional approach. Although various approaches for the renormalization have been proposed, our approach is useful to improve the accuracy for the numerical results of the RTE.

Acknowledgements This work was partially supported by JSPS KAKENHI Grant Numbers 15K17980, 15K06125, and 25287028; and by Japan Agency for Medical Research and Development. 


\section{References}

1. Balsara, D.: Fast and accurate discrete ordinates methods for multidimensional radiative transfer . Part I , basic methods. J. Quant. Spectrosc. Radiat. Transfer 69, 671-707 (2001)

2. Boulet, P., Collin, A., Consalvi, J.L.: On the finite volume method and the discrete ordinates method regarding radiative heat transfer in acute forward anisotropic scattering media. J. Quant. Spectrosc. Radiat. Transfer 104, 460-473 (2007)

3. Carlson, B.G.: Quadrature Tables of Equal Weight EQn Over the Unit sphere. Los Alamos Scientific Laboratory Report 4734 (1971)

4. Chandrasekhar, S.: Radiative Transfer. Dover, New York (1960)

5. Cheong, W.F., Prahl, S.A., Welch, A.J.: A review of the optical properties of biological tissue. IEEE J. Quantum Electron 26(12), 2166-2185 (1990)

6. Fiveland, W.A.: The selection of discrete ordinate quadrature sets for anisotropic scattering. ASME, HTD-vol. 160, Fundamentals of radiation heat transfer pp. 89-96 (1991)

7. Fujii, H., Okawa, S., Nadamoto, K., Okada, E., Yamada, Y., Hoshi, Y., Watanabe, M.: Numerical modeling of photon migration in the cerebral cortex of the living rat using the radiative transport equation. Proc. SPIE 2015 9333, 933313 1-5 (2015)

8. Fujii, H., Okawa, S., Nadamoto, K., Okada, E., Yamada, Y., Hoshi, Y., Watanabe, M.: Numerical modeling of photon migration in human neck based on the radiative transport equation. Journal of Applied Nonlinear Dynamics 5(1), 117-125 (2016) eprint arXiv:1603.03769 [physics.med-ph]

9. Fujii, H., Okawa, S., Yamada, Y., Hoshi, Y.: Hybrid model of light propagation in random media based on the time-dependent radiative transfer and diffusion equations. J. Quant. Spectrosc. Radiat. Transfer 147, 145-154 (2014)

10. Gibson, A.P., Hebden, J.C., Arridge, S.R.: Recent advances in diffuse optical imaging. Phys. Med. Biol. 50(4), R1-R43 (2005)

11. Gottlieb, S., Shu, C.W.: Total variation diminishing Runge-Kutta schemes. Math. Comp. 67(221), 73-85 (1998)

12. Han, W., Eichholz, J.A., Wang, G.: On a family of differential approximations of the radiative transfer equation. J. Math. Chem. 50, 689-702 (2012).

13. Henyey, L.G., Greenstein, L.J.: Diffuse radiation in the galaxy. J. Astrophys. 93, 70-83 (1941)

14. Hunter, B., Guo, Z.: Conservation of asymmetry factor in phase function discretization for radiative transfer analysis in anisotropic scattering media. Int. J. Heat Mass Transfer 55, 1544-1552 (2012)

15. Joseph, J.H., Wiscombe, W.J., Weinman, J.A.: The delta-Eddington approximation for radioactive flux transfer. J. Atmos. Sci. 33, 2452-2459 (1976)

16. Klose, A.D., Netz, U., Beuthan, J., Hielscher, A.H.: Optical tomography using the timeindependent equation of radiative transfer - Part 1 : forward model. J. Quant. Spectrosc. Radiat. Transfer 72, 691-713 (2002)

17. Liu, L., Ruan, L., Tan, H.: On the discrete ordinates method for radiative heat transfer in anisotropically scattering media. Int. J. Heat Mass Transfer 45(15), 3259-3262 (2002)

18. Martelli, F., Sassaroli, A., Pifferi, A., Torricelli, A., Spinelli, L., Zaccanti, G.: Heuristic Green's function of the time dependent radiative transfer equation for a semi-infinite medium. Opt. Express 15(26), 18,168-18,175 (2007)

19. Montejo, L.D., Klose, A.D., Hielscher, A.H.: Implementation of the equation of radiative transfer on block-structured grids for modeling light propagation in tissue. Biomed. Opt. Express 1(3), 861-878 (2010)

20. Mori, M., Sugihara, M.: The double-exponential transformation in numerical analysis. J. Comput. Appl. Math. 127, 287-296 (2001)

21. Ning, H., Simos, T.E..: A low computational cost eight algebraic order hybrid method with vanished phase-lag and its first, second, third and fourth derivatives for the approximate solution of the Schrödinger equation. J. Math. Chem. 53(6), 1295-1312 (2015)

22. Paasschens, J.C.J.: Solution of the time-dependent Boltzmann equation. Phys. Rev. E 56(1), 1135-1141 (1997)

23. Papadopoulos, D.F., Simos, T.E.: A modified Runge-Kutta-Nyström method by using phase lag properties for the numerical solution of orbital problems. Appl. Math. Inf. Sci. 7(2), 433-437 (2013)

24. Simos, T.E.: Closed Newton-Cotes trigonometrically-fitted formulae of high order for long-time integration of orbital problems. Appl. Math. Lett. 22, 1616-1621 (2009) 
25. Takahasi, H., Mori, M.: Double exponential formulas for numerical indefinite integration. Publ. RIMS Kyoto Univ. 9, 721-741 (1974)

26. Zheng, H., Han, W.: On simplified spherical harmonics equations for the radiative transfer equation. J. Math. Chem. 49, 1785-1797 (2011) 